\title{
MEAN VALUE INEQUALITIES IN HILBERT SPACE
}

\author{
F. H. CLARKE AND YU. S. LEDYAEV
}

\begin{abstract}
We establish a new mean value theorem applicable to lower semicontinuous functions on Hilbert space. A novel feature of the result is its "multidirectionality": it compares the value of a function at a point to its values on a set. We then discuss some refinements and consequences of the theorem, including applications to calculus, flow invariance, and generalized solutions to partial differential equations.
\end{abstract}

RÉsumé. On établit un nouveau théorème de la valeur moyenne qui s'applique aux fonctions semicontinues inférieurement sur un espace de Hilbert. On déduit plusieurs conséquences du résultat portant, par exemple, sur les fonctions monotones et sur les solutions généralisées des équations aux dérivées partielles.

\section{INTRODUCTION}

The central theme of this article is the estimation of functional value differences via differential information at intermediate points. The classical model for such results is the mean value theorem of calculus, or its progenitor, the mean value inequality:

$$
f(y)-f(x) \leq\left\langle f^{\prime}(z), y-x\right\rangle,
$$

where $z$ is on the line segment determined by $x$ and $y$. This result has undergone considerable generalization. In this article we introduce what appears to be a new consideration: multidirectionality.

Specifically, we estimate the differences $f(y)-f(x)$, where now $y$ ranges over a set $Y$. When $f$ is a smooth function on $\mathbb{R}^{n}$, for example, our main theorem asserts the existence of a point $z$ in the "interval" $[x, Y]$ (i.e., the convex hull of $\{x\} \cup Y$ ) such that

$$
\min _{Y} f-f(x) \leq\left\langle f^{\prime}(z), y-x\right\rangle \quad \forall y \in Y .
$$

The theorem is developed in a nonsmooth setting, however, and in the context of a Hilbert space, both factors being important for the purposes of applications. We are not aware of results in the literature of comparable nature, in either the smooth or nonsmooth cases.

Received by the editors January 15, 1993 and, in revised form, June 21, 1993.

1991 Mathematics Subject Classification. Primary 26B05.

Key words and phrases. Mean value theorem, nonsmooth analysis, flow invariance, monotone functions, generalized solutions of partial differential equations.

The support of the Natural Sciences and Engineering Research Council of Canada and of le Fonds FCAR (Québec) is gratefully acknowledged. 
Section 2 is devoted to a statement and proof of our main result (Theorem 2.1). The following section deduces its "infinitesimal" versions, in particular an extension and strengthening of a theorem of A.I. Subbotin, whose work was the original stimulus for ours. The remaining sections can be read in any order. Section 4 obtains a refinement of the theorem under a stronger regularity assumption. Section 5 presents an application to the invariance theory of differential inclusions, and Section 6 develops a new type of functional monotonicity and a corresponding criterion for it. Section 7 compares function values on two sets. In the final section, we introduce two new definitions of generalized solution of a first-order partial differential equation. One of these is an extension to Hilbert space of Subbotin's original "minimax solution" concept, which does not appear to be sufficiently well-known, and the other ("proximal solution") is new. As an application of our results, we prove that these solution concepts are equivalent to one another, as well as to that of "viscosity solution".

Let us establish some notation: $H$ is a Hilbert space, $\langle\cdot, \cdot\rangle$ its inner product, $\|\cdot\|$ its norm, and $B$ the closed unit ball in $H$. If $x$ is a point and $Y$ a set in $H,[x, Y]$ refers to the set $\{z: z=x+t(y-x)$ for some $t \in[0,1]$ and $y \in Y\}$. We shall work with an extended-valued function $f: H \rightarrow(-\infty, \infty]$, always assumed lower semicontinuous. A proximal subgradient of $f$ at $x$, where $x$ is a point at which $f$ is finite, is an element $\zeta$ of $H$ satisfying the following condition: for some $\sigma \geq 0$, and for all $y$ in some neighborhood of $x$, we have

$$
f(y)-f(x)+\sigma\|y-x\|^{2} \geq\langle\zeta, y-x\rangle .
$$

The set of proximal subgradients of $f$ at $x$ (which may very well be empty) is denoted $\partial^{\pi} f(x)$; if $f$ admits a (Gâteaux or Fréchet) derivative $f^{\prime}(x)$ at $x$, the only possible proximal subgradient of $f$ at $x$ is $\zeta=f^{\prime}(x)$, but $f^{\prime}(x)$ may not be a proximal subgradient. We remark that $\partial^{\pi} f(x)$ is a basic building block of nonsmooth analysis [2], but that no elements of that theory will be called upon. In fact, the article is completely self-contained, with the exception of the use of the Borwein-Preiss variational principle, which we state in Section 2.

\section{THE MAIN RESULT}

Let $H$ be a Hilbert space, and let $f: H \rightarrow(-\infty, \infty]$ be a lower semicontinuous extended-valued function. We wish to relate the value of $f$ at a given point $x$ at which $f$ is finite to the values of $f$ on a subset $Y$ of $H$. We assume throughout that $Y$ is nonempty, closed, bounded and convex.

Let

$$
\hat{r}(Y, x):=\lim _{\delta \downarrow 0} \inf _{y \in Y+\delta B}\{f(y)-f(x)\},
$$

where $\hat{r}(Y, x)$ is naturally interpreted to be $+\infty$ if $f=+\infty$ everywhere in $Y+\delta B$ for some $\delta>0$. (Note that $\hat{r}(Y, x)$ coincides with $\min _{Y} f-f(x)$ if $Y$ is compact, in particular when $H=\mathbb{R}^{n}$.)

Theorem 2.1. Let $f$ be bounded below on $[x, Y]+\delta B$ for some $\delta>0$. Then for any $r<\hat{r}(Y, x)$ and $\epsilon>0$ there exist $z \in[x, Y]+\epsilon B$ and $\zeta \in \partial^{\pi} f(z)$ such that

$$
r<\langle\zeta, y-x\rangle \text { for all } y \in Y \text {. }
$$


Further, we can choose $z$ to satisfy $f(z)<\inf _{[x, Y]} f+|r|+\epsilon$.

The following is obtained by an evident limiting procedure as $\epsilon \rightarrow 0$ in the theorem.

Corollary 2.1. In addition to the hypotheses of the theorem, suppose that $Y$ is compact, and that $f$ is continuously Fréchet differentiable in a neighborhood of $[x, Y]$. Then there exists a point $z \in[x, Y]$ such that

$$
f(z) \leq \min _{[x, Y]} f+|\hat{r}(Y, x)|,
$$

and

$$
\min _{Y} f-f(x) \leq\left\langle f^{\prime}(z), y-x\right\rangle \text { for all } y \in Y .
$$

Remark 2.1. A form of Corollary 2.1 can be proven for Banach spaces, and for the case in which the point $x$ is replaced by a set $X$ [3], by using techniques quite different from those employed here, and which do not apply in the context of merely lower semicontinuous functions.

The following is a more specific instance of Corollary 2.1, obtained by taking $Y=x+\delta B$.

Corollary 2.2. Let $f$ be continuously differentiable on $\mathbb{R}^{n}$, and let $\delta>0$. Then there exists $z \in x+\delta B$ with $f(z) \leq f(x)$ such that

$$
\min _{y \in x+\delta B} f(y) \leq f(x)-\delta\left|f^{\prime}(z)\right|
$$

Before actually proving the theorem, we sketch the motivating idea behind it; this digression can be skipped without any loss of continuity. Consider minimizing

$$
(t, y) \rightarrow f(x+t(y-x))-r t
$$

over $(t, y) \in[0,1] \times Y$, and suppose a minimum exists at $(\bar{t}, \bar{y})$. If $\bar{t}$ is interior to $[0,1]$ and if $f$ is smooth, then the existence of a local minimum at $\bar{t}$ (for $y=\bar{y}$ fixed) yields

$$
\left\langle\bar{y}-x, f^{\prime}(z)\right\rangle=r,
$$

where $z:=x+\bar{t} \bar{y}$. The minimum over $Y$ with respect to the $y$ variable gives

$$
\left\langle\bar{t} f^{\prime}(z), y-\bar{y}\right\rangle \geq 0 \quad \forall y \in Y .
$$

Together, these two conclusions imply

$$
\left\langle f^{\prime}(z), y-x\right\rangle \geq r \quad \forall y \in Y,
$$

which is more or less the required conclusion. The actual proof must deal with noninterior $\bar{t}$, the fact that $f$ is nonsmooth, and the possible nonexistence of minima.

Proof of Theorem 2.1. We shall denote $[x, Y]+\delta B$ by $V$. We may assume without loss of generality that $x=0$ and that $\epsilon<\delta$. Fix $\bar{r}$ in the open interval $(r, \min \{\hat{r}(Y, x), r+\epsilon / 2\})$ and define, for any positive integer $k$, a function $f_{k}$ on $H \times H$ as follows:

$$
f_{k}(y, v):=f(v)+k\|y-v\|^{2} .
$$


We also define a function $\varphi_{k}$ on $[0,1]$ via

$$
\begin{aligned}
\varphi_{k}(t) & :=\inf _{(y, v) \in Y \times V}\left\{f_{k}(t y, v)-\bar{r} t\right\} \\
& =\inf _{(y, v) \in Y \times V}\left\{f(v)+k\|t y-v\|^{2}-\bar{r} t\right\} .
\end{aligned}
$$

Note that $\varphi_{k}(t)$ is finite for each $t$ and $k$ (in particular since $f(0)$ is finite). Because $Y$ and $V$ are bounded, it is easy to verify that $\varphi_{k}$ is continuous. Let $t_{k}$ be a minimizer of $\varphi_{k}$ on $[0,1]$.

Lemma 1. For $k$ sufficiently large, we have $t_{k}<1$.

To prove the lemma it suffices to show that for $k$ sufficiently large,

$$
\varphi_{k}(1)>\varphi_{k}(0) \text {. }
$$

Suppose to the contrary that $\varphi_{k_{i}}(1) \leq \varphi_{k_{i}}(0)$ for some sequence $k_{i} \rightarrow \infty$, and let $\left(y_{i}, v_{i}\right)$ in $Y \times V$ satisfy

$$
f\left(v_{i}\right)+k_{i}\left\|y_{i}-v_{i}\right\|^{2}-\bar{r} \leq \varphi_{k_{i}}(0)+\frac{1}{k_{i}} .
$$

Since $\varphi_{k_{i}}(0) \leq f(0)$ (take $v=0$ in the definition of $\varphi_{k_{i}}(0)$ ), and since by hypothesis $f \geq-L$ on $V$ for some $L>0$, we deduce

$$
\left\|v_{i}-y_{i}\right\| \leq \delta_{i}
$$

where $\delta_{i}:=C k_{i}^{-1 / 2}$ and

$$
C:=(L+|f(0)|+|\bar{r}|+1)^{1 / 2} .
$$

This implies that $d\left(v_{i}, Y\right) \leq \delta_{i}$, and hence in light of (2.2) that

$$
\inf _{y \in Y+\delta_{i} B}\{f(y)-f(0)\} \leq f\left(v_{i}\right)-f(0) \leq \varphi_{k_{i}}(0)-f(0)+\bar{r}+\frac{1}{k_{i}} \leq \bar{r}+\frac{1}{k_{i}} .
$$

But the extreme left side of this inequality converges to $\hat{r}(Y, 0)$ as $i \rightarrow \infty$, which contradicts the choice of $\bar{r}$. This proves Lemma 1 .

Lemma 2. Let $\left(y^{\prime}, v^{\prime}\right)$ in $Y \times V$ satisfy

$$
f_{k}\left(t_{k} y^{\prime}, v^{\prime}\right)<\inf _{(y, v) \in Y \times V} f_{k}\left(t_{k} y, v\right)+\frac{1}{k} .
$$

Then

$$
d\left(v^{\prime},[0, Y]\right) \leq C / k^{1 / 2} .
$$

To see this, note first that

$$
\begin{aligned}
& \inf _{(y, v) \in Y \times V} f_{k}\left(t_{k} y, v\right)=\inf _{(y, v) \in Y \times V}\left\{f_{k}\left(t_{k} y, v\right)-\bar{r} t_{k}\right\}+\bar{r} t_{k} \\
& \left.\quad=\inf _{t \in[0,1]} \inf _{(y, v) \in Y \times V}\left\{f_{k}(t y, v)-\bar{r} t\right\}+\bar{r} t_{k} \quad \text { (by definition of } t_{k}\right) \\
& \quad \leq f_{k}(0,0)+\bar{r} t_{k} \leq f(0)+|\bar{r}| .
\end{aligned}
$$

Thus if $\left(y^{\prime}, v^{\prime}\right)$ satisfies (2.3) we then have

$$
k\left\|v^{\prime}-t_{k} y^{\prime}\right\|^{2} \leq L+|f(0)|+|\bar{r}| \leq C^{2} .
$$

Since $t_{k} y^{\prime}$ belongs to $[0, Y]$, this implies (2.4). 
Now let us fix a value of $k$ so that the right side of (2.4) (and thus, $1 / k$ too) is less than $\epsilon / 2$, and so that $t_{k}<1$ (see Lemma 1). The proof proceeds via two cases, each employing a sequence $\epsilon_{i}$ decreasing to 0 , with $0<\epsilon_{i}^{3}<1 / k \forall i$.

Case 1: $t_{k}>0$. For each $i$, there is a pair $\left(y_{i}, v_{i}\right) \in Y \times V$ satisfying

$$
f_{k}\left(t_{k} y_{i}, v_{i}\right) \leq \inf _{(y, v) \in Y \times V} f_{k}\left(t_{k} y, v\right)+\epsilon_{i}^{3} .
$$

In view of Lemma 2 we have $d\left(v_{i},[0, Y]\right)<\epsilon$.

Consider now the minimization of the function $\tilde{f}(y, v):=f_{k}\left(t_{k} y, v\right)$ over $(y, v) \in Y \times V$. Then $\left(y_{i}, v_{i}\right)$ "almost solves" this problem, which may not actually admit an exact solution. A version of the Borwein-Preiss variational principle will allow us to draw a conclusion.

Theorem (Borwein-Preiss [1]). Let $M$ be a closed subset of a Hilbert space $E$, and let $g: E \rightarrow(-\infty, \infty]$ be lower semicontinuous and bounded below on $M$. For some $\epsilon^{\prime}>0$ and $x_{0} \in M$, let

$$
g\left(x_{0}\right)<\inf _{M} g+\epsilon^{\prime} .
$$

Then for any $\lambda>0$ there exist $\bar{w} \in M$ and $\tilde{u} \in E$ such that

$$
\left\|x_{0}-\bar{w}\right\|<\lambda, \quad\|\bar{w}-\tilde{u}\|<\lambda, \quad g(\bar{w})<\inf _{M} g+\epsilon^{\prime},
$$

and for any $x \in M, x \neq \bar{w}$, we have

$$
g(\bar{w})+\frac{\epsilon^{\prime}}{\lambda^{2}}\|\bar{w}-\tilde{u}\|^{2}<g(x)+\frac{\epsilon^{\prime}}{\lambda^{2}}\|x-\tilde{u}\|^{2} .
$$

We apply this theorem for the function $\tilde{f}$ defined above on $E:=H \times H$, with $M=Y \times V, \epsilon^{\prime}=\epsilon_{i}^{3}, \lambda=\epsilon_{i}, x_{0}=\left(y_{i}, v_{i}\right)$. We obtain the existence of pairs $\left(\bar{y}_{i}, \bar{v}_{i}\right) \in Y \times V,\left(\tilde{y}_{i}, \tilde{v}_{i}\right) \in H \times H$ such that

$$
\begin{aligned}
\left\|y_{i}-\bar{y}_{i}\right\|<\epsilon_{i},\left\|v_{i}-\bar{v}_{i}\right\| & <\epsilon_{i},\left\|\bar{y}_{i}-\tilde{y}_{i}\right\|<\epsilon_{i},\left\|\bar{v}_{i}-\tilde{v}_{i}\right\|<\epsilon_{i} . \\
f_{k}\left(t_{k} \bar{y}_{i}, \bar{v}_{i}\right) & <\inf _{Y \times V} f_{k}\left(t_{k} y, v\right)+\epsilon_{i}^{3},
\end{aligned}
$$

and such that the pair $\left(\bar{y}_{i}, \bar{v}_{i}\right)$ minimizes the function

$$
\tilde{g}(y, v):=f(v)+k\left\|t_{k} y-v\right\|^{2}+\epsilon_{i}\left(\left\|v-\tilde{v}_{i}\right\|^{2}+\left\|y-\tilde{y}_{i}\right\|^{2}\right)
$$

over the set $Y \times V$.

In view of Lemma 2, we derive from (2.7) that $\bar{v}_{i}$ is less than distance $\delta$ from the set $[0, Y]$ (recall that we arranged to have $C / k^{1 / 2}<\epsilon<\delta$ ), so that $\bar{v}_{i}$ lies in the interior of $V(=[0, Y]+\delta B)$.

Lemma 3. The vector $\zeta_{i}=2 k\left(t_{k} \bar{y}_{i}-\bar{v}_{i}\right)+2 \epsilon_{i}\left(\tilde{v}_{i}-\bar{v}_{i}\right)$ satisfies $\zeta_{i} \in \partial^{\pi} f\left(\bar{v}_{i}\right)$.

To see this, we note that $\tilde{g}\left(\bar{y}_{i}, \cdot\right)$ attains a local minimum at $\bar{v}_{i}$, which gives, for $v$ near $\bar{v}_{i}$ :

$$
f(v)-f\left(\bar{v}_{i}\right) \geq k\left(\left\|t_{k} \bar{y}_{i}-\bar{v}_{i}\right\|^{2}-\left\|t_{k} \bar{y}_{i}-v\right\|^{2}\right)+\epsilon_{i}\left(\left\|\tilde{v}_{i}-\bar{v}_{i}\right\|^{2}-\left\|\tilde{v}_{i}-v\right\|^{2}\right) .
$$

Rearranging leads to

$$
f(v)-f\left(\bar{v}_{i}\right) \geq\left\langle\zeta_{i}, v-\bar{v}_{i}\right\rangle-2\left(k+\epsilon_{i}\right)\left\|v-\bar{v}_{i}\right\|^{2},
$$

which gives the required conclusion by definition of $\partial^{\pi} f$. 
We require some estimates concerning the function $\varphi_{k}$ :

$$
\begin{aligned}
\varphi_{k}\left(t_{k}\right) & =\inf _{Y \times V}\left\{f(v)+k\left\|t_{k} y-v\right\|^{2}-\bar{r} t_{k}\right\} \\
& \geq f_{k}\left(t_{k} \bar{y}_{i}, \bar{v}_{i}\right)-\epsilon_{i}^{3}-\bar{r} t_{k} \quad(\text { in view of }(2.7)) \\
& =f\left(\bar{v}_{i}\right)+k\left\|t_{k} \bar{y}_{i}-\bar{v}_{i}\right\|^{2}-\epsilon_{i}^{3}-\bar{r} t_{k},
\end{aligned}
$$

and

$$
\varphi_{k}\left(t_{k}+\epsilon_{i}\right) \leq f\left(\bar{v}_{i}\right)+k\left\|\left(t_{k}+\epsilon_{i}\right) \bar{y}_{i}-\bar{v}_{i}\right\|^{2}-\bar{r}\left(t_{k}+\epsilon_{i}\right) .
$$

Using these and the fact that $\varphi_{k}$ is minimized over $[0,1]$ at $t_{k}<1$, we calculate

$$
\begin{aligned}
0 & \leq \liminf _{i \rightarrow \infty} \frac{\varphi_{k}\left(t_{k}+\epsilon_{i}\right)-\varphi_{k}\left(t_{k}\right)}{\epsilon_{i}} \\
& \leq \liminf _{i \rightarrow \infty}\left\{2 k\left\langle\bar{y}_{i},\left(t_{k}+\epsilon_{i} / 2\right) \bar{y}_{i}-\bar{v}_{i}\right\rangle-\bar{r}+\epsilon_{i}^{2}\right\} \\
& =-\bar{r}+\liminf _{i \rightarrow \infty}\left\langle\zeta_{i}, \bar{y}_{i}\right\rangle
\end{aligned}
$$

(where (2.6) has been used).

That is, we have

$$
\liminf _{i \rightarrow \infty}\left\langle\zeta_{i}, \bar{y}_{i}\right\rangle \geq \bar{r}
$$

Now we use the fact that $\bar{y}_{i}$ minimizes the quadratic function $\tilde{g}\left(\cdot, \bar{v}_{i}\right)$ over the convex set $Y$. The necessary (and sufficient) condition for this is

$$
\left\langle 2 k t_{k}\left(t_{k} \bar{y}_{i}-\bar{v}_{i}\right)+2 \epsilon_{i}\left(\bar{y}_{i}-\tilde{y}_{i}\right), y-\bar{y}_{i}\right\rangle \geq 0 \quad \forall y \in Y .
$$

Because $t_{k}>0$ (the assumption defining Case 1), and since $Y$ is bounded and (2.6) holds, this implies, for some constant $\sigma$ not depending on $i$ or $y$,

$$
\left\langle\zeta_{i}, y-\bar{y}_{i}\right\rangle+\sigma \epsilon_{i} \geq 0 \quad \forall y \in Y .
$$

Combining (2.8) and (2.9), and bearing in mind that $\bar{r}>r$, we conclude that for $i$ sufficiently large,

$$
\left\langle\zeta_{i}, y\right\rangle>r \quad \forall y \in Y,
$$

which proves the theorem, with $\zeta=\zeta_{i}$ and $z=\bar{v}_{i}$, except for the upper bound on $f(z)$. But in view of (2.7) we have

$$
\begin{aligned}
f(z) & =f\left(\bar{v}_{i}\right)<\inf _{Y \times V}\left\{f_{k}\left(t_{k} y, v\right)-\bar{r} t_{k}\right\}+\bar{r} t_{k}+\epsilon_{i}^{3}=\min _{[0,1]} \varphi_{k}(t)+\bar{r} t_{k}+\epsilon_{i}^{3} \\
& \leq \inf _{[0,1] \times Y}\{f(t y)-\bar{r} t\}+\max (0, \bar{r})+\epsilon / 2 \\
& \leq \inf _{[0, Y]} f+\max (0,-\bar{r})+\max (0, \bar{r})+\epsilon / 2 \\
& =\inf _{[0, Y]} f+|\bar{r}|+\epsilon / 2 \leq \inf _{[0, Y]} f+|r|+\epsilon .
\end{aligned}
$$

Case 2: $t_{k}=0$. Let $v_{i} \in V$ satisfy

$$
f_{k}\left(0, v_{i}\right)<\inf _{V} f_{k}(0, v)+\epsilon_{i}^{3} .
$$

Appealing to the Borwein-Preiss theorem, we get the existence of points $\bar{v}_{i} \in V$ and $\tilde{v}_{i} \in H$ such that

$$
\begin{array}{r}
\left\|v_{i}-\bar{v}_{i}\right\|<\epsilon_{i},\left\|\bar{v}_{i}-\tilde{v}_{i}\right\|<\epsilon_{i}, \\
f_{k}\left(0, \bar{v}_{i}\right)<\inf _{V} f_{k}(0, v)+\epsilon_{i}^{3},
\end{array}
$$


and such that $\bar{v}_{i}$ minimizes on $V$ the function

$$
\tilde{g}(v):=f_{k}(0, v)+\epsilon_{i}\left\|v-\tilde{v}_{i}\right\|^{2} .
$$

We deduce from Lemma 2 that $\bar{v}_{i}$ is an interior point of $V$. As before, the vector $\zeta_{i}$ of Lemma 3 (with $t_{k}$ now equal to zero) belongs to $\partial^{\pi} f\left(\bar{v}_{i}\right)$.

We note the following estimates, for any $y \in Y$ :

$$
f_{k}\left(0, \bar{v}_{i}\right)-\epsilon_{i}^{3} \leq \varphi_{k}(0) \leq \varphi_{k}\left(\epsilon_{i}\right) \leq f_{k}\left(\epsilon_{i} y, \bar{v}_{i}\right)-\epsilon_{i} \bar{r}
$$

This implies

$$
0 \leq k\left\|\epsilon_{i} y-\bar{v}_{i}\right\|^{2}-k\left\|\bar{v}_{i}\right\|^{2}-\epsilon_{i} \bar{r}+\epsilon_{i}^{2} .
$$

Upon dividing across by $\epsilon_{i}$, the term on the right is expressible in the form

$$
2 k\left\langle y,\left(\epsilon_{i} / 2\right) y-\bar{v}_{i}\right\rangle-\bar{r}+\epsilon_{i} .
$$

The first term of this expression is bounded above by $\left\langle\zeta_{i}, y\right\rangle+\sigma \epsilon_{i}$ for some constant $\sigma$ not depending on $i$ or $y$. Since $r<\bar{r}$, we deduce that for $i$ large enough, (2.10) holds. The upper bound on $f(z)$ is proven just as in Case 1, with the help of (2.11).

This completes the proof.

Remark 2.2. The proof shows that in the statement of the theorem, $[x, Y]+\epsilon B$ can be replaced by any closed bounded set $V$ which contains $[x, Y]+\epsilon B$ for some $\epsilon>0$, and on which $f$ is bounded below.

\section{INFINITESIMAL VERSIONS À LA SUbbotin}

For a given subset $E$ of $H$ and a point $x$ at which the function $f$ is finite, we introduce $\underline{D} f(x ; E)$, the (possibly infinite) quantity given by

$$
\liminf _{t \downarrow 0, \delta \downarrow 0} \inf _{e \in E+\delta B} \frac{f(x+t e)-f(x)}{t} .
$$

This reduces to a more familiar Dini derivative when $E$ is a singleton $\{e\}$ :

$$
\underline{D}^{s} f(x ; e):=\liminf _{t \downarrow 0, e^{\prime} \rightarrow e} \frac{f\left(x+t e^{\prime}\right)-f(x)}{t},
$$

where $e^{\prime} \rightarrow e$ indicates norm convergence and the "s" in $\underline{D}^{s}$ signifies "strong". Theorem 3.1. Let $E$ be a nonempty closed, bounded, convex subset of $H$, and let $f: H \rightarrow(-\infty, \infty]$ be lower semicontinuous, and finite at a point $x$. Suppose for some scalar $\rho$ we have

$$
\underline{D} f(x ; E)>\rho .
$$

Then for any $\epsilon^{\prime}>0$ there exist a point $z$ and $\zeta \in \partial^{\pi} f(z)$ such that

$$
\|z-x\|<\epsilon^{\prime}, \quad|f(z)-f(x)|<\epsilon^{\prime}
$$

and

$$
\langle\zeta, e\rangle>\rho \quad \forall e \in E .
$$

Proof. Fix $\epsilon^{\prime}>0$ and $\bar{\rho} \in(\rho, \underline{D} f(x ; E))$; we may assume $\epsilon^{\prime 2}<2 \epsilon^{\prime}$ without loss of generality. It follows from (3.1) and the lower semicontinuity of $f$ that there are $\delta \in\left(0, \epsilon^{\prime}\right), \tau>0$ such that

$$
\tau E \subset \frac{\delta}{2} B, \quad 2|\bar{\rho}| \tau<\epsilon^{\prime}
$$


and such that, for any $e \in E+\delta B, t \in[0, \tau], x^{\prime} \in x+\delta B$ we have

$$
f(x+t e)>f(x)+t \bar{\rho}, f\left(x^{\prime}\right)>f(x)-\epsilon^{\prime} .
$$

Let $Y=x+\tau E$; then the first relation in (3.3) yields $\hat{r}(Y, x) \geq \tau \bar{\rho}=: \bar{r}$. We apply Theorem 2.1 with $r=\tau \rho, \epsilon=\epsilon^{\prime} / 2$, to deduce the existence of $z \in[x, Y]+(\epsilon / 2) B$ and $\zeta \in \partial^{\pi} f(z)$ such that

$$
\langle\zeta, \tau e\rangle>\tau \rho \quad \forall e \in E,
$$

which establishes (3.2). We also derive $\|z-x\|<\epsilon^{\prime}$ from the way in which $\delta$ and $\tau$ were specified.

In order to obtain the final conclusion regarding $f(z)$, we will invoke $\operatorname{Re}$ mark 2.2 and refer to the proof of Theorem 2.1, which is valid for $V=x+\delta B$. The point $z$ produced by the proof is of the form $\bar{v}_{i}$ (for some $i$ ), where $\bar{v}_{i}$ minimizes the function $\tilde{g}\left(\bar{y}_{i}, \cdot\right)$ over $V$. We have

$$
\begin{aligned}
f(z)-\tau \bar{\rho} t_{k}-\epsilon_{i}^{3} & \leq f(z)+k\left\|t_{k} \bar{y}_{i}-\bar{v}_{i}\right\|^{2}-\bar{r} t_{k}-\epsilon_{i}^{3} \\
& =f_{k}\left(t_{k} \bar{y}_{i}, \bar{v}_{i}\right)-\bar{r} t_{k}-\epsilon_{i}^{3} \\
& \leq \inf _{Y \times V} f_{k}\left(t_{k} y, v\right)-\bar{r} t_{k} \\
& =\varphi_{k}\left(t_{k}\right) \leq \varphi_{k}(0) \\
& \leq f(x)
\end{aligned}
$$

Thus

$$
f(z) \leq f(x)+\epsilon_{i}^{3}+\tau|\bar{\rho}|
$$

But $k$ and $\epsilon_{i}^{3}$ were chosen to satisfy

$$
\epsilon_{i}^{2}<\frac{1}{k}<\frac{C^{2}}{k}<\epsilon^{2}=\left(\frac{\epsilon^{\prime}}{2}\right)^{2}
$$

which is less than $\epsilon^{\prime} / 2$ by assumption. In addition, $\tau|\bar{\rho}|$ is bounded above by $\epsilon^{\prime} / 2$, and so the upper bound just derived for $f(z)$, together with the second part of (3.3), yields

$$
|f(z)-f(x)|<\epsilon^{\prime}
$$

as required.

We now introduce a new sequential weak lower Dini derivative

$$
\underline{D}^{w} f(x ; e):=\inf _{\left\{e_{i}\right\}} \lim _{t \downarrow 0} \inf _{e_{i} \rightarrow e} \frac{f\left(x+t e_{i}\right)-f(x)}{t},
$$

where $e_{i} \stackrel{w}{\rightarrow} e$ denotes weak convergence, and where the infimum is taken over all sequences $\left\{e_{i}\right\}$ converging weakly to $e$.

Proposition 3.1. If $E$ is a nonempty, closed, bounded, convex subset of $H$, then

$$
\inf _{e \in E} \underline{D}^{w} f(x ; e) \leq \underline{D} f(x ; E) .
$$

Proof. Let $t_{i}, \delta_{i}, e_{i}$ be sequences such that $t_{i} \downarrow 0, \delta_{i} \downarrow 0, e_{i} \in E+\delta_{i} B$, and

$$
\frac{f\left(x+t_{i} e_{i}\right)-f(x)}{t_{i}} \rightarrow \underline{D} f(x ; E) .
$$


We may take a subsequence (without relabeling) to arrange $e_{i} \stackrel{w}{\rightarrow} e \in E$. Then

$$
\underline{D}^{w} f(x ; e) \leq \underline{D} f(x ; E) .
$$

The following extends an important result of Subbotin [9] to infinite dimensions, and also strengthens it in the finite-dimensional case in several respects: the use of $\partial^{\pi} f$ constitutes a refinement, and the values of $f$ are kept close to one another.

Corollary 3.1. Let $E$ be a nonempty, closed, bounded, convex subset of $H$, and let $f: H \rightarrow(-\infty, \infty]$ be a lower semicontinuous function, finite at a point $x$. Suppose that for some $\rho$ we have

$$
\inf _{e \in E} \underline{D}^{w} f(x ; e)>\rho .
$$

Then for any $\epsilon>0$ there exist $z$ and $\zeta \in \partial^{\pi} f(z)$ such that $\|z-x\|<$ $\epsilon,|f(z)-f(x)|<\epsilon$, and (3.2) holds.

Proof. In view of Proposition 3.1, (3.5) implies (3.1), so Theorem 3.1 applies directly.

Remark 3.1. When $H=\mathbb{R}^{n}$, the strong and weak lower Dini derivatives coincide, the infimum in (3.4) is attained, and equality holds. A slightly stronger form of Theorem 3.1 is then true: $\underline{D} f(x ; E)$ can be replaced by

$$
\liminf _{t \downarrow 0, e \in E} \frac{f(x+t e)-f(x)}{t}
$$

(we obtain this by restricting $e$ to $E$ rather than to $E+\delta B$ in the proof).

Remark 3.2. The convexity of $E$ cannot be deleted from the hypotheses: consider $f(u)=\|u\|$ on $\mathbb{R}^{2}$, with $x=0$ and $E$ the unit sphere. We may take $\rho=1 / 2$, and (3.2) then asserts

$$
\langle\zeta, e\rangle>1 / 2 \quad \forall e \in E,
$$

which is impossible.

\section{A REFINEMENT IN THE DIFFERENTIABLE CASE}

Theorem 2.1 does not assert that the point $z$ lies in $[x, Y]$, which may seem surprising. Here is an example to illustrate how $z$ may have to lie outside $[x, Y]$ in general. Set $H=\mathbb{R}^{1}, x=0, Y=\{1\}$, and define

$$
f(u)= \begin{cases}-\sqrt{|u|} & \text { for } u \leq 0, \\ 1 & \text { for } u>0 .\end{cases}
$$

Then $\hat{r}(Y, x)=1$, and applying the theorem for $r=1 / 2$ gives a point $z$ and $\zeta \in \partial^{\pi} f(z)$ such that $\zeta>1 / 2$. But $\partial^{\pi} f(z)=\{0\}$ if $z>0$, and $\partial^{\pi} f(0)=\varnothing$, so $z$ necessarily lies outside $[0,1]$.

When $f$ is differentiable, however, we can refine the theorem as follows.

Theorem 4.1. Let $f: H \rightarrow(-\infty, \infty]$ be lower semicontinuous, and finite at $x$, and let $Y$ be a nonempty, convex, closed, bounded subset of $H$. Suppose in addition that $f$ is Fréchet differentiable and bounded below on $[x, Y]$. Then for any $r$ satisfying

$$
r<\inf _{Y} f-f(x)
$$


and for any $\epsilon>0$, there exist $z$ in $[x, Y]$ such that

$$
r<\left\langle f^{\prime}(z), y-x\right\rangle \quad \forall y \in Y,
$$

and

$$
f(z)<\inf _{[x, Y]} f+|r|+\epsilon .
$$

Proof. We may assume $x=0$. Choose $\bar{r}$ in $(r, r+\epsilon / 2)$ with

$$
r<\bar{r}<\inf _{Y} f-f(0),
$$

and define $\varphi(t, y)=f(t y)-\bar{r} t$, a function which is lower semicontinuous and bounded below on $[0,1] \times Y$. Let $\epsilon_{i}$ be a sequence decreasing to 0 , and let $\left(t_{i}, y_{i}\right)$ be a pair in $[0,1] \times Y$ satisfying

$$
\varphi\left(t_{i}, y_{i}\right)<\inf _{[0,1] \times Y} \varphi+\epsilon_{i}^{3} .
$$

We appeal to the Borwein-Preiss theorem to deduce the existence of $\left(\bar{t}_{i}, \bar{y}_{i}\right) \in$ $[0,1] \times Y,\left(\tilde{t}_{i}, \tilde{y}_{i}\right) \in \mathbb{R} \times H$ such that

(4.1) $\left|t_{i}-\bar{t}_{i}\right|<\epsilon_{i}, \quad\left\|y_{i}-\bar{y}_{i} \quad\right\|<\epsilon_{i},\left|\bar{t}_{i}-\tilde{t}_{i}\right|<\epsilon_{i}, \quad\left\|\bar{y}_{i}-\tilde{y}_{i}\right\|<\epsilon_{i}$,

$$
\varphi\left(\bar{t}_{i}, \bar{y}_{i}\right)<\inf _{[0,1] \times Y} \varphi+\epsilon_{i}^{3}
$$

and such that the function

$$
\tilde{g}(t, y):=\varphi(t, y)+\epsilon_{i}\left(\left|t-\tilde{t}_{i}\right|^{2}+\left\|y-\tilde{y}_{i}\right\|^{2}\right)
$$

attains a minimum over $[0,1] \times Y$ at $\left(\bar{t}_{i}, \bar{y}_{i}\right)$.

We claim that for $i$ sufficiently large, $\bar{t}_{i}<1$. If not, then for arbitrarily large $i$ we have

$$
\tilde{g}\left(1, \bar{y}_{i}\right) \leq \tilde{g}\left(0, \tilde{y}_{i}\right)
$$

But

$$
\tilde{g}\left(1, \bar{y}_{i}\right) \geq f\left(\bar{y}_{i}\right)-\bar{r}
$$

and

$$
\tilde{g}\left(0, \tilde{y}_{i}\right) \leq f(0)+\epsilon_{i},
$$

and hence

$$
\inf _{Y} f-\bar{r} \leq f(0)+\epsilon_{i}
$$

which contradicts the choice of $\bar{r}$ for $i$ large.

Now let us suppose that for indices $i$ arbitrarily large, we have

$$
1>\bar{t}_{i}>\epsilon_{i} \text {. }
$$

Then for such $i$, for any $y \in Y$, for all $\lambda>0$ sufficiently small, the vector

$$
y^{\lambda}:=\bar{y}_{i}+\lambda\left(y-\bar{y}_{i}\right) / \bar{t}_{i}
$$

belongs to $Y$ (since $Y$ is convex). Thus

$$
\tilde{g}\left(\bar{t}_{i}+\lambda, y^{\lambda}\right)-\tilde{g}\left(\bar{t}_{i}, \bar{y}_{i}\right) \geq 0 .
$$


Dividing this inequality by $\lambda$ and letting $\lambda$ decrease to 0 gives

$$
\left\langle f^{\prime}\left(\bar{t}_{i} \bar{y}_{i}\right), y\right\rangle-\bar{r}+\alpha_{i} \geq 0,
$$

where

$$
\alpha_{i}=2 \epsilon_{i}\left\{\left\langle\bar{y}_{i}-\tilde{y}_{i}, y-\bar{y}_{i}\right\rangle / \bar{t}_{i}+\left(\bar{t}_{i}-\tilde{t}_{i}\right)\right\} .
$$

It follows from (4.1) and (4.3) and the boundedness of $Y$ that for some constant $D$ depending only on $Y$, and not on $i$ or $y$, one has $\left|\alpha_{i}\right| \leq D \epsilon_{i}$. Thus when $i$ is large enough, (4.4) gives the conclusion of the theorem (for $z=\bar{t}_{i} \bar{y}_{i}$ ), except for the upper bound on $f(z)$. But in view of (4.2) we have

$f\left(\bar{t}_{i} \bar{y}_{i}\right)<\inf _{[0,1] \times Y}\{f(t y)-\bar{r} t\}+\bar{r} \bar{t}_{i}+\epsilon_{i}^{3} \leq \inf _{[0, Y]} f+\max (0,-\bar{r})+\max (0, \bar{r})+\epsilon_{i}^{3}$,

and the right side is less than

$$
\inf _{[x, Y]} f+|r|+\epsilon
$$

for $i$ large, as required.

Now let us deal with the case in which, for all $i$ large,

$$
\bar{t}_{i} \leq \epsilon_{i} \text {. }
$$

Then for all $y \in Y$ and $\lambda \in[0,1 / 2]$, for all $i$ large,

$$
\tilde{g}\left(\bar{t}_{i}+\lambda, y\right)-\tilde{g}\left(\bar{t}_{i}, \bar{y}_{i}\right) \geq 0 .
$$

Set $\lambda=\epsilon_{i}^{1 / 2}$; then in light of (4.5) and Fréchet differentiability we derive

$$
\begin{aligned}
\tilde{g}\left(\bar{t}_{i}+\lambda, y\right) & =f(0)+\epsilon_{i}^{1 / 2}\left\{\left\langle f^{\prime}(0), y\right\rangle-\bar{r}\right\}+o\left(\epsilon_{i}^{1 / 2}\right), \\
\tilde{g}\left(\bar{t}_{i}, \bar{y}_{i}\right) & =f(0)+o\left(\epsilon_{i}^{1 / 2}\right),
\end{aligned}
$$

where

$$
\lim _{i \rightarrow \infty} o\left(\epsilon_{i}^{1 / 2}\right) / \epsilon_{i}^{1 / 2}=0
$$

uniformly for $y \in Y$. Substituting in (4.6), dividing by $\epsilon_{i}^{1 / 2}$ and going to the limit leads to $\left\langle f^{\prime}(0), y\right\rangle \geq \bar{r}$, which proves the theorem (for $z=0$; the upper bound on $f(z)$ follows as before).

Remark 4.1. When $\inf _{Y} f>f(x)$, then $x \notin Y$, and it follows that the point $z$ can be asserted to lie outside of $Y$. For then we can choose $r$ and $\epsilon$ without loss of generality to satisfy

$$
0<r=\inf _{Y} f-f(x)-\epsilon,
$$

and the upper bound on $f(z)$ given by the theorem reduces to

$$
f(z)<\inf _{[x, Y]} f-f(x)+\inf _{Y} f \leq \inf _{Y} f,
$$

which ensures $z \notin Y$.

Finally we remark that even in finite dimensions when $f$ is continuously differentiable, it is not possible in general to assert that $z$ lies in the relative 
interior of $[x, Y]$ (as in the classical case when $Y$ is a singleton); see Example 2 of [3].

\section{INVARIANCE OF DIFFERENTIAL INCLUSIONS}

Consider the differential inclusion

$$
\dot{x}(t) \in F(x(t)) \text { a.e. },
$$

where the multifunction $F$ maps $\mathbb{R}^{n}$ into the closed convex subsets of $\mathbb{R}^{n}$ and is upper semicontinuous. Let $K$ be a closed subset of $\mathbb{R}^{n}$. $K$ is said to be weakly invariant for (5.1) if for every $x_{0} \in K$ there exists an absolutely continuous solution $x(\cdot)$ of $(5.1)$ such that $x(0)=x_{0}$ and $x(t) \in K$ for $t \geq 0$.

A well-known necessary and sufficient condition for $K$ to be weakly invariant is the following:

$$
F(x) \cap T_{K}(x) \neq \varnothing \text { for all } x \in K,
$$

where $T_{K}(x)$ is the contingent tangent cone to $K$ at $x$ :

$$
T_{K}(x)=\left\{e: \liminf _{t \downarrow 0} \frac{d_{K}(x+t e)}{t}=0\right\},
$$

$d_{K}(\cdot)$ being the Euclidean distance function associated with $K$.

Subbotin et al. [6] have shown that an equivalent criterion for weak invariance is the apparently weaker requirement

$$
F(x) \cap \operatorname{co} T_{K}(x) \neq \varnothing \text { for all } x \in K .
$$

We give now a new simple proof of this equivalence based on the results of section 4.

Theorem 5.1. Conditions (5.2) and (5.3) are equivalent.

Proof. We suppose that (5.3) holds, but that for some $x \in K$, we have

$$
F(x) \cap T_{K}(x)=\varnothing .
$$

Then for some $\delta>0$ we have

$$
(F(x)+\delta B) \cap T_{K}(x)=\varnothing,
$$

where $B$ is the closed unit ball. Set $E:=F(x)+\delta B$. Then (5.4) and the definition of $T_{K}$ imply

$$
\liminf _{t \downarrow 0} \inf _{e \in E} \frac{\psi_{K}(x+t e)}{t}>0
$$

where $\psi_{K}$ is the indicator function of $K$ (i.e., $\psi_{K}=0$ on $K$ and $+\infty$ elsewhere). We apply Corollary 3.1 to $f=\psi_{K}$ to deduce for any $\epsilon>0$ the existence of a point $z$ in $x+\epsilon B$, with $z \in K$ (since the $f$ values are close), and $\zeta \in \partial^{\pi} \psi_{K}(z)$ such that

$$
\langle\zeta, v\rangle>0 \quad \forall v \in F(x)+\delta B .
$$

For $\epsilon$ small enough, $F(x)+\delta B$ contains $F(z)$, whence

$$
\langle\zeta, v\rangle>0 \quad \forall v \in F(z) \text {. }
$$

We have, by definition of $\partial^{\pi} \psi_{K}$,

$$
\langle\zeta, y-z\rangle \leq \sigma|y-z|^{2} \text { for all } y \in K,
$$


which can easily be seen to imply

$$
\zeta \cdot e \leq 0 \text { for all } e \in T_{K}(z),
$$

i.e., $\zeta \in T_{K}(x)^{*}$, the polar of $T_{K}(z)$.

Now since (5.3) holds, there exists $\hat{v} \in F(z)$ such that

$$
\langle\xi, \hat{v}\rangle \leq 0 \text { for all } \xi \in T_{K}(z)^{*},
$$

and in particular when $\xi=\zeta$. Thus

$$
\langle\zeta, \hat{v}\rangle \leq 0
$$

which contradicts (5.5).

\section{A MONOTONICITY RESULT}

The mean value theorem has been an important tool in the development of calculus, both in the classical theory and in the nonsmooth setting (see Loewen [7] for a discussion of mean value theorems in nonsmooth analysis). In this tradition, we give in this section an application to calculus of the multidirectional mean value inequality, one which appears to be new even in the smooth case. The systematic use of the multidirectional mean value inequality to develop calculus will be carried out elsewhere.

Let $D$ be a nonempty, compact, convex subset of $H$, and let $f: H \rightarrow$ $(-\infty, \infty]$ be lower semicontinuous.

Theorem 6.1. Suppose that

$$
u \in H, \zeta \in \partial^{\pi} f(u) \Rightarrow \min _{d \in D}\langle\zeta, d\rangle \leq 0 .
$$

Then, for any $x$, and for any $t>0$, we have

$$
\min _{y \in x+t D} f(y) \leq f(x)
$$

Remark 6.1. When $D$ is a singleton $\{d\}$, the conclusion of the theorem admits the usual interpretation of monotonicity in the $d$-direction:

$$
f(x+t d) \leq f(x)
$$

(where both sides can equal $+\infty$ ). Otherwise, the theorem transforms the hypothesis of instantaneous (i.e. proximal) nonincrease for some $d$ in $D$ (depending on the point) into a global conclusion (which we could call "weak monotonicity") bearing upon any $x$ and its translates by the set $D$. When $f$ is smooth, a proof of the result can be based on trajectories generated by "directions of descent" (or nonascent), a recourse not available in the less regular setting.

Remark 6.2. A different sort of "strong monotonicity" result has been proven in [4]. The hypothesis there is more demanding:

$$
\max _{d \in D}\langle\zeta, d\rangle \leq 0
$$

(rather than " $\min$ "), and the conclusion is correspondingly stronger:

$$
\max _{y \in x+t D} f(y) \leq f(x) \text {. }
$$


This follows easily from the unidirectional type of mean value theorem, since one can study one direction at a time to get the required conclusion. In contrast, the "weak monotonicity" result of Theorem 6.1 illustrates well the deeper level of information inherent in the multidirectional mean value inequality.

Proof of Theorem 6.1. We assume $f(x)$ is finite, for otherwise there is nothing to prove. We seek to apply Theorem 2.1 , with $Y:=x+t D$. We have

$$
\hat{r}(Y, x)=\min _{y \in x+t D} f(y)-f(x),
$$

and we now choose any $r<\hat{r}(Y, x)$. It suffices to show that $r \leq 0$. But Theorem 2.1 asserts that for some $z$, and for some $\zeta \in \partial^{\pi} f(z)$,

$$
r<\min _{d \in D}\langle\zeta, d\rangle,
$$

and the right side is nonpositive by hypothesis.

\section{Comparing FUnCtion VAlUes ON TWO SETS}

Let $X$ and $Y$ be compact subsets of $H$, and suppose that we have

$$
f(x)<0, \quad f(y)>0 \quad \forall x \in X, \forall y \in Y .
$$

If $f$ is smooth, it follows from the classical mean value theorem that for any given $(x, y) \in X \times Y$, there is a point $z$ on the line segment $[x, y]$ such that

$$
\left\langle f^{\prime}(z), y-x\right\rangle>0 \text {. }
$$

We proceed to obtain a uniform version of such a conclusion, which appears to be new.

We shall require the following notion. (A cone is a set closed under positive scalar multiplication, and cone $(C)$ signifies the smallest cone containing a given set $C$.)

Definition 7.1. The point $p$ is said to be a focal point for the sets $X$ and $Y$ if cone $(Y-X)=$ cone $(Y-\{p\})$ and cone $(X-Y)=\operatorname{cone}(X-\{p\})$.

As a guide to intuition, we remark that if $X$ and $Y$ are the opposite faces of a parallelogram in $\mathbb{R}^{2}$, then the unique focal point $p$ is the point at which the diagonals intersect. It appears that in $\mathbb{R}^{2}$, when $X$ and $Y$ are disjoint compact, convex sets, there is always a unique focal point; in higher dimensions it is easy to see that such points $p$ may or may not exist.

Theorem 7.1. Let $f: H \rightarrow(-\infty, \infty)$ be continuous and satisfy (7.1), where $X, Y$ are compact convex subsets of $H$ admitting a focal point $p$. Suppose that $f$ is Fréchet differentiable on the set

$$
\Omega:=[p, X] \cup[p, Y] .
$$

Then there exists $z$ in $\Omega$ such that

$$
\left\langle f^{\prime}(z), y-x\right\rangle>0 \quad \forall x \in X, \quad \forall y \in Y .
$$

Proof. We argue two cases, depending on the value of $f(p)$. Let $\Delta>0$ be such that $f \leq-\Delta$ on $X$, and $f \geq \Delta$ on $Y$. 
Case 1. : $f(p)<\Delta$ In this case, we shall apply Theorem 4.1 for $x=p, Y=Y$. Note that $\lambda:=\min _{Y} f-f(p)$ is positive; choose $r$ in $(0, \lambda)$. The theorem then asserts the existence of $z$ in $[p, Y]$ such that one has

$$
\left\langle f^{\prime}(z), y^{\prime}-p\right\rangle>r>0 \quad \forall y^{\prime} \in Y .
$$

Let $(x, y)$ be any pair in $X \times Y$. Then

$$
y-x \in \operatorname{cone}(Y-X)=\operatorname{cone}(Y-\{p\}) .
$$

Hence for some $y^{\prime} \in Y$ and $t>0$, one has $y-x=t\left(y^{\prime}-p\right)$. But then (7.3) implies (7.2).

Case 2: $\quad \Delta \leq f(p)$. We can apply essentially the same argument as in Case 1, with the role of $Y$ now played by $X$, and with $-f$ for $f$; we omit the details.

Example 7.1. In $\mathbb{R}^{n}$, let $X$ and $Y$ be opposite faces of a unit cube:

$$
\begin{aligned}
& X=\left\{\left(0, u_{2}, u_{3}, \ldots, u_{n}\right): 0 \leq u_{i} \leq 1, i=2,3, \ldots, n\right\}, \\
& Y=\left\{\left(1, u_{2}, u_{3}, \ldots, u_{n}\right): 0 \leq u_{i} \leq 1, i=2,3, \ldots, n\right\} .
\end{aligned}
$$

Then $X$ and $Y$ admit the focal point

$$
p=(1 / 2,1 / 2, \ldots, 1 / 2) .
$$

If $f$ is negative on $X$ and positive on $Y$ (and differentiable), the theorem asserts that for some point $z$ in the set $[p, X] \cup[p, Y]$ one has

$$
f_{1}^{\prime}-\left|f_{2}^{\prime}\right|-\cdots-\left|f_{n}^{\prime}\right|>0,
$$

where the partial derivatives $f_{i}^{\prime}$ are evaluated at $z$.

A different approach to the two-set case is developed in [3].

\section{GeNERALIZED SOlUtions OF FIRST-ORDER PARTIAL DIFFERENTIAL EQUATIONS}

Let $F: H \times \mathbb{R} \times H \rightarrow \mathbb{R}$ be a continuous function, and let $\Omega$ be an open subset of the Hilbert space $H$. Our purpose in this section is to discuss generalized solutions of the partial differential equation

$$
F(x, u(x), \nabla u(x))=0, \quad x \in \Omega .
$$

One such concept is that of viscosity solution [5].

Definition 8.1. A lower semicontinuous function $u: H \rightarrow(-\infty, \infty$ ] [an upper semicontinuous function $v: H \rightarrow[-\infty, \infty)]$ is said to be a viscosity supersolution [subsolution] of $(8.1)$ if it satisfies the following condition: whenever there is a point $x \in \Omega$ at which the function is finite and a function $\varphi$ Fréchet differentiable at $x$ such that $u-\varphi$ attains a local minimum $[v-\varphi$ attains a local maximum] at $x$, then relation (8.2) [(8.3)] below holds:

$$
\begin{aligned}
& F(x, u(x), \nabla \varphi(x)) \geq 0 \\
& F(x, v(x), \nabla \varphi(x)) \leq 0 .
\end{aligned}
$$

(This varies slightly from the terminology in [5] by allowing semisolutions to be extended-valued.) A viscosity solution of (8.1) is a continuous function which is simultaneously a viscosity supersolution and subsolution.

It is natural to introduce another definition in more intrinsic terms. 
Definition 8.2. A lower semicontinuous function $u: H \rightarrow(-\infty, \infty$ ] [an upper semicontinuous function $v: H \rightarrow[-\infty, \infty)]$ is said to be a proximal supersolution [subsolution] of $(8.1)$ if relation $(8.4)[(8.5)]$ holds:

$$
\begin{array}{ll}
F(x, u(x), \zeta) \geq 0 \quad \forall \zeta \in \partial^{\pi} u(x), & \forall x \in \Omega \\
F(x, v(x), \zeta) \leq 0 \quad \forall \zeta \in \partial_{\pi} v(x), & \forall x \in \Omega .
\end{array}
$$

(Here, $\partial_{\pi} v(x)$ signifies the proximal supergradient of $v$ at $x$ : the set $-\partial^{\pi}(-v)(x)$.) Again, a proximal solution of (8.1) is a continuous function which is both a proximal subsolution and supersolution.

One of the earliest notions of generalized solutions originated in the work of Subbotin in differential games [8], [10], [11] (in finite dimensions). We give here a simplified version of his definition adapted to infinite dimensions; it turns out to be a faithful extension.

Definition 8.3. A lower semicontinuous function $u: H \rightarrow(-\infty, \infty$ ] [an upper semicontinuous function $v: H \rightarrow[-\infty, \infty)]$ is said to be a minimax supersolution [subsolution] of (8.1) if relation (8.6) [(8.7)] holds for any $x \in \Omega$ at which the function is finite:

$$
\begin{aligned}
& \sup _{p \in H} \inf _{v \in H}\left\{\underline{D}^{w} u(x ; v)-\langle p, v\rangle-F(x, u(x), p)\right\} \leq 0, \\
& \inf _{p \in H} \sup _{v \in H}\left\{\bar{D}^{w} u(x ; v)-\langle p, v\rangle-F(x, u(x), p)\right\} \geq 0 .
\end{aligned}
$$

(Here, $\underline{D}^{w}$ is the Dini derivative defined in Section 3, while $\bar{D}^{w}$ is the analogous derivative in which "lim inf" is replaced by "limsup".) A minimax solution of $(8.1)$ is a continuous function which is both a minimax subsolution and supersolution.

We use below the following Lipschitz hypothesis on $F$.

$$
\left\{\begin{array}{l}
\text { There exists a locally bounded function } K: \Omega \times \mathbb{R} \rightarrow[0, \infty) \\
\text { such that for all }(x, \alpha) \in \Omega \times \mathbb{R}, \text { for all } p \text { and } q \text { in } H, \\
|F(x, \alpha, p)-F(x, \alpha, q)| \leq K(x, \alpha)\|p-q\| .
\end{array}\right.
$$

Theorem 8.1. Let $F$ be continuous and satisfy $(\mathrm{H})$, and let $w: \Omega \rightarrow \mathbb{R}$ be continuous. Then the following are equivalent:

(a) $w$ is a minimax solution;

(b) $w$ is a viscosity solution;

(c) $w$ is a proximal solution.

Proof. We shall actually prove a little more: if $u: \Omega \rightarrow(-\infty, \infty]$ is a lower semicontinuous function, then the following are equivalent:

(a) ${ }^{\prime} \quad u$ is a minimax supersolution;

(b) ${ }^{\prime} \quad u$ is a viscosity supersolution;

(c) ${ }^{\prime} \quad u$ is a proximal supersolution.

This, together with the analogous result for subsolutions, gives the theorem. The implications $(a)^{\prime} \Rightarrow(b)^{\prime} \Rightarrow(c)^{\prime}$ are easy, and will be done first. The implication $(\mathrm{c})^{\prime} \Rightarrow(\mathrm{a})^{\prime}$ will follow from the results of Section 3. 
Suppose then that $u$ is a minimax supersolution. Let $x$ be a point in $\Omega$ at which $u$ is finite and $\varphi$ a function differentiable at $x$ such that $u-\varphi$ has a local minimum at $x$. Then, for any $v \in H$,

$$
0 \leq \underline{D}^{w}(u-\varphi)(x ; v)=\underline{D}^{w} u(x ; v)-\langle\nabla \varphi(x), v\rangle .
$$

According to (8.6) (identify $p$ with $\nabla \varphi(x)$ ), for any $\epsilon>0$ there exists $v$ such that

$$
\underline{D}^{w} u(x ; v)-\langle\nabla \varphi(x), v\rangle-F(x, u(\dot{x}), \nabla \varphi(x)) \leq \epsilon .
$$

But then, in light of (8.8), we have

$$
F(x, u(x), \nabla \varphi(x)) \geq-\epsilon .
$$

Since $\epsilon$ is arbitrary, we conclude that (8.2) holds, and so that $u$ is a viscosity supersolution.

Now let $u$ be a viscosity supersolution, and let $\zeta$ belong to $\partial^{\pi} u(x)$ for some $x \in \Omega$. Then from the definition of $\partial^{\pi} u(x)$, the function $u-\varphi$ has a (finite) local minimum at $x$, where $\varphi$ is defined by

$$
\varphi(y):=\langle\zeta, y-x\rangle-\sigma\|y-x\|^{2}
$$

for some $\sigma \geq 0$. Since $\nabla \varphi(x)=\zeta$, we deduce from (8.2)

$$
F(x, u(x), \zeta) \geq 0,
$$

which confirms (8.4) and the fact that $u$ is a proximal subsolution.

Suppose now that $u$ is a proximal supersolution, but fails to be a minimax supersolution. We will derive a contradiction, which establishes $(\mathrm{c})^{\prime} \Rightarrow(\mathrm{a})^{\prime}$ and completes the proof. Since $u$ is not a minimax supersolution, there exists $x_{0} \in \Omega, p \in H$, and $\Delta>0$ such that $u\left(x_{0}\right)$ is finite and

$$
\underline{D}^{w} u\left(x_{0} ; v\right)-\langle p, v\rangle-F\left(x_{0}, u\left(x_{0}\right), p\right) \geq 2 \Delta \quad \forall v \in H .
$$

Let $M$ be a number such that

$$
K(x, \alpha) \leq M \text { for all }(x, \alpha) \text { near }\left(x_{0}, u\left(x_{0}\right)\right) .
$$

We may write, in view of (8.9),

$$
\underline{D}^{w} f\left(x_{0} ; v\right) \geq 2 \Delta+F\left(x_{0}, u\left(x_{0}\right), p\right) \quad \forall v \text { with }\|v\| \leq M,
$$

where $f$ is the function $f(x):=u(x)-\langle p, x\rangle$. We proceed to invoke Corollary 3.1 for a decreasing sequence $\epsilon_{i}$ tending to 0 to deduce the existence for each $i$ of points $x_{i}$ and vectors $\zeta_{i} \in \partial^{\pi} u\left(x_{i}\right)$ such that

$$
\begin{gathered}
\left\|x_{i}-x_{0}\right\|<\epsilon_{i}, \quad\left|u\left(x_{i}\right)-u\left(x_{0}\right)+\left\langle p, x_{0}-x_{i}\right\rangle\right|<\epsilon_{i}, \\
\left\langle\zeta_{i}-p, v\right\rangle \geq \Delta+F\left(x_{0}, u\left(x_{0}\right), p\right) \text { if }\|v\| \leq M .
\end{gathered}
$$

It follows that $\left(x_{i}, u\left(x_{i}\right)\right)$ converges to $\left(x_{0}, u\left(x_{0}\right)\right)$, so that for $i$ large we have

$$
F\left(x_{i}, u\left(x_{i}\right), p\right) \leq F\left(x_{0}, u\left(x_{0}\right), p\right)+\Delta / 2 .
$$

We now invoke hypothesis $(\mathrm{H})$ to deduce that when $i$ is large enough for both (8.10) and (8.12) to apply, then

$$
\begin{aligned}
F\left(x_{i}, u\left(x_{i}\right), \zeta_{i}\right) & \leq F\left(x_{i}, u\left(x_{i}\right), p\right)+M\left\|\zeta_{i}-p\right\| \\
& \leq F\left(x_{0}, u\left(x_{0}\right), p\right)+\Delta / 2-\left\langle\zeta_{i}-p, v\right\rangle
\end{aligned}
$$

(for some $v$ of norm $M$ ) 


$$
\leq-\Delta / 2 \quad(\text { by }(8.11)) \text {. }
$$

But in view of (8.4), we must have

$$
F\left(x_{i}, u\left(x_{i}\right), \zeta_{i}\right) \geq 0 .
$$

This contradiction completes the proof.

Remark 8.1. The hypothesis (H) was used only in the proof of the last implication. In finite dimensions, it can be dispensed with altogether, and in infinite dimensions it can be replaced by a weaker uniform continuity condition. We omit these details here, since the present intention is merely to underline the application of the earlier results of the article. They will appear with other related developments in a subsequent article.

\section{REFERENCES}

1. J. M. Borwein and D. Preiss, A smooth variational principle with applications to subdifferentiability and to differentiability of convex functions, Trans. Amer. Math. Soc. 303 (1987), 517-527.

2. F. H. Clarke, Methods of dynamic and nonsmooth optimization, CBMS-NSF Regional Conf. Ser. in Appl. Math., vol. 57, SIAM, Philadelphia, Pa., 1989.

3. F. H. Clarke and Yu. S. Ledyaev, Mean value inequalities, Proc. Amer. Math. Soc. (to appear).

4. F. H. Clarke, R. J. Stern and P. R. Wolenski, Subgradient criteria for monotonicity, the Lipschitz condition, and convexity, Canad. J. Math. 45 (1993), 1167-1183.

5. M. G. Crandall, H. Ishii and P. L. Lions, User's guide to viscosity solutions of second order partial differential equations, Bull. Amer. Math. Soc. 27 (1992), 1-67.

6. H. G. Guseinov, A. I. Subbotin and V. N. Ushakov, Derivatives for multivalued mappings with applications to game-theoretical problems of control, Problems Control Inform. Theory 14 (1985), 155-167.

7. P. D. Loewen, Optimal control via nonsmooth analysis, CRM Proc. \& Lecture Notes, vol. 2, Amer. Math. Soc., Providence, R.I., 1993.

8. A. I. Subbotin, A generalization of the basic equation of the theory of differential games, Soviet Math. Dokl. 22 (1980), 358-362.

9. __ On a property of a subdifferential, Mat. Sb. 1982 (1991), 1315-1330. (Russian)

10. __ Continuous and discontinuous solutions of boundary value problems for first-order partial differential equations, Dokl. Akad. Nauk SSSR 323 (1992), no. 2. (Russian)

11. _ Viable characteristics of Hamilton-Jacobi equations, preprint.

Centre de Recherches Mathématiques, Université de Montréal, C.P. 6128-A, Montréal, Québec H3C 3J7, Canada

Steklov Mathematics Institute, Vavilov St. 42, Moscow 117966, Russia 